\title{
"BASLEENDER
} Trascender, Contabilidad y Gestión Núm. 11 (mayo - agosto del 2019). ISSN: 2448-6388. Universidad de Sonora. Departamento de Contabilidad. Reserva de Derechos 04-2015-04172070800-203.

\section{Auditoría al desempeño: evolución y relevancia en el ámbito estatal}

Performance audit: evolution and relevance at the state level

$$
\text { Jesús Antonio Valenzuela Bojórquez }{ }^{1}
$$

\section{Resumen}

La auditoría al desempeño, es de relevancia porque permite verificar el cumplimiento de objetivos y metas con indicadores estratégicos y de gestión, para conocer los resultados de la aplicación de los recursos públicos y el impacto social que se genera a través de diversos programas y proyectos; por lo que promueve la gobernanza económica, eficaz y eficiente y contribuye a la rendición de cuentas y a la transparencia.

El objetivo en este trabajo es evaluar la evolución y relevancia que presenta en el ámbito estatal, con referencia del nivel federal las auditorías al desempeño (AD) realizadas por el Instituto Superior de Auditoría y Fiscalización (ISAF) y por la Secretaría de la Contraloría General (SECOG) con entes que han sido objeto de fiscalización en el estado de Sonora; esto, a través de un trabajo de tipo documental y descriptivo. El análisis de los datos se abordó desde un aspecto cualitativo y cuantitativo. Se exponen resultados obtenidos en las entidades gubernamentales en los aspectos sustantivos y críticos de su misión, resaltando que a nivel nacional la Auditoría Superior de la Federación (ASF) en el transcurso de catorce años, realizó solamente veintidós AD en Sonora. A nivel local estatal, los entes fiscalizadores están en etapa de gestación en este tipo de auditoría; las cifras oficiales reportan inicio de la AD en el ejercicio 2018; a la vez, se aporta un instrumento de apoyo para practicar auditorías al desempeño, al ser escasa o nulamente conocidos en los procesos del ámbito estatal.

Palabras clave: auditoría al desempeño, sector público, metas.

DOI: https://doi.org/10.36791/tcg.v11i0.66

JEL: H83 Administración pública Contabilidad y auditorías del sector público.

Recibido: 25 de noviembre de 2018.

Aceptado: 20 de abril de 2019.

\footnotetext{
${ }^{1}$ Jesús Antonio Valenzuela Bojórquez. Contador Público. Supervisor de Auditoría en el Órgano Interno de Control de la Secretaría de la Contraloría General (SCG). Correo: antonio.valenzuela.bojorquez@gmail.com
} 


\begin{abstract}
The performance audit is relevant because it allows to verify the achievement of objectives and goals with strategic and management indicators, to know the results of the application of public resources and the social impact that is generated through various programs and projects; Therefore, it promotes economic, effective and efficient governance and contributes to accountability and transparency.
\end{abstract}

The objective in this work is to evaluate the evolution and relevance that it presents in the state level, with reference of the federal performance audits made by Instituto Superior de Auditoría y Fiscalización (ISAF) and by Secretaría de la Contraloría General (SECOG) with entities that have been subject to supervision in the state of Sonora; this, through a documentary and descriptive type of work. The analysis of the data was approached from a qualitative and quantitative aspect. Results obtained in the governmental entities are exposed in the substantive and critical aspects of their mission, highlighting that at national level Auditoría Superior de la Federación (ASF) in the course of fourteen years, made only twenty-two ADs in Sonora. At the local state level, the auditing bodies are in the stage of gestation in this type of audit; the official figures report the beginning of the $\mathrm{AD}$ in 2018; At the same time, a support instrument is provided to practice performance audits, as they are limited or not known in the processes of the state.

Keywords: performance audit, public sector, goals. 


\section{Introducción}

En la gestión gubernamental los resultados pueden medirse en términos cuantitativos y, en especial, también en términos cualitativos, haciendo énfasis en los impactos económicos y sobre todo sociales de los diferentes programas que utilizan recursos públicos. Por lo tanto, mediante las AD puede medirse el grado de satisfacción ciudadana respecto a la gestión de un programa, lo cual es un objetivo social de todo ente gubernamental.

En la gestión gubernamental, las actividades deben ser medidas y evaluadas, esta situación obedece a que los recursos no son propios y hay el deber legal de informar cómo se obtienen, cómo se administran, en qué se ejercen, cuáles objetivos y metas se alcanzan. Por tal motivo, el gobierno necesita evaluar su desempeño y calificar la precisión de su planeación estratégica, así como combatir la corrupción y la impunidad al constituir una obligación constitucional.

Las auditorías al desempeño deben realizarse por mandato constitucional según lo disponen los artículos 74 y 79 de nuestra Constitución Política, el primero haciendo referencia a las facultades de revisión de la Cámara de Diputados; y el segundo, señalando que dichas facultades se realizarán mediante un Órgano autónomo denominado Auditoría Superior de la Federación.

En el ámbito estatal de igual forma existe un mandato constitucional para efectuar auditorías al desempeño, y el artículo 67 de la constitución estatal señala que el Instituto Superior de Auditoría y Fiscalización, se constituye como un órgano del Congreso del Estado y estará encargado de la revisión y fiscalización de los estados financieros $\mathrm{y}$ cuentas públicas estatales $\mathrm{y}$ municipales.

Este trabajo adquiere un interés especial por varias razones:
- Los trabajos de auditoría relacionados con auditorías al desempeño en el ámbito gubernamental estatal de Sonora, son de reciente aplicación, debido a que fue a partir de ejercicio 2018 cuando se implementaron en el Instituto Superior de Auditoría y Fiscalización (ISAF) y en la Secretaría de la Contraloría General (SECOG).

- El tema requiere de un estudio minucioso y ordenado para identificar claramente los medios idóneos que se requieren para desarrollar una auditoría al desempeño.

- Responde a problemáticas reales que presentan las entidades gubernamentales, las cuales pocas veces son detectadas e informadas para buscar soluciones y oportunidades de mejora.

Las AD otorgan información, análisis y perspectivas sobre el quehacer gubernamental para minimizar los costos de los recursos empleados; obtener el máximo de los insumos disponibles; lograr los resultados previstos, y verificar el impacto social y económico para la ciudadanía; permitiendo así evaluar la operación de los programas con base en economía, eficiencia y eficacia, alineando sus resultados con los objetivos gubernamentales.

Esta investigación se compone de los siguientes apartados: primeramente, se abordan las situaciones relacionadas con los antecedentes de la función de fiscalización y auditoría en su ámbito general, después se detalla el problema de investigación, relacionado con el tema auditoría al desempeño en el estado de Sonora. Se realiza una justificación del tema de estudio y se exponen los objetivos generales y específicos que se desean desarrollar. Posteriormente, se desarrolla el planteamiento metodológico de la investigación, señalando el método, enfoque, tipo de investigación, alcance, ámbito geográfico donde se va a desarrollar el trabajo, lo sitios visitados para la recolección de datos, así como los problemas y limitaciones para el desarrollo del tema. 
También se muestra el marco referencial, plasmando algunos resultados obtenidos por investigadores en varias partes del mundo. Asimismo, se aborda el marco conceptual, detallando algunos conceptos básicos. Se presenta una breve descripción del principal marco jurídico (legal) de las AD. También se citan los diferentes entes que pueden y están facultados para realizar revisiones o fiscalizaciones en el ámbito federal, estatal y municipal. Por último, se presenta un plan de trabajo para ayudar a las personas interesadas en el desarrollo de este tipo de auditorías al desempeño, siendo este plan únicamente de carácter enunciativo, pudiendo adaptarse y perfeccionarse según las circunstancias requeridas al momento de la revisión. (Dicho programa se muestra en el anexo 1 al final del trabajo).

Por lo antepuesto, objetivo de esta investigación radica en la importancia de elaborar auditorías al desempeño (AD), logrando así exponer los resultados obtenidos por las entidades gubernamentales estatales de Sonora en los aspectos críticos y sustantivos de su misión.

\section{Antecedentes}

Desde que existe el hombre, debido a su capacidad de raciocinio ha desarrollado una tendencia a controlar el medio ambiente que le rodea, por lo tanto, diseñaba controles de sus actividades primarias, como son la agricultura, ganadería, pesca, vestido, lo cual era realizado por intuición y experiencia, sin definir un concepto a dichas actividades; sin embargo, aunque en términos rudimentarios, el objetivo era controlar los riesgos, es decir, era una similitud a lo que en la actualidad se conoce como auditoría. "El Código de Hammurabi de Babilonia es el primer documento legal conocido por el hombre, en donde se maneja el concepto de contaduría forense, la cual consiste en demostrar con documentación contable un fraude o una mentira” (Leon \& Lagunas, 2017, p. 13).
Derivado de la importancia de los resultados que se obtienen de la implementación de la $\mathrm{AD}$, en otros países esta auditoría tiene varios años de aplicación, según publicación del Banco Interamericano de Desarrollo Jarquin, Molina \& Roseth (2018, p. 2-3) señalan que Estados Unidos encabeza la lista con un historial de más de nueve décadas, desde 1921, cuando la US GAO obtuvo el mandato a tal efecto, Suecia tiene el mandato a partir de 1967; Holanda, desde 1976; Gran Bretaña desde 1983; Noruega desde 2004 y Nueva Zelanda desde 2001.

El 31 de marzo de 2008, en México, fue publicado en el Diario Oficial de la Federación, el Acuerdo por el que se establecen las disposiciones generales del Sistema de Evaluación del Desempeño (SED), siendo el principal objetivo verificar el cumplimiento de objetivos y metas, para lo cual se diseñaron indicadores estratégicos y de gestión. Con lo anterior, se pretende conocer los resultados de la aplicación de los recursos federales, el impacto social de los programas y de los proyectos en la Administración Pública Federal.

Con fecha 7 de mayo de 2008, derivado de la necesidad de rendir cuentas a la sociedad, el Congreso de la Unión, realizó varias reformas constitucionales enfocadas a medir los objetivos, metas y resultados obtenidos en la gestión gubernamental; para lo cual se reformó el artículo 134 constitucional.

El 31 de diciembre de 2008, se crea en México la Ley General de Contabilidad Gubernamental (LGCG), la cual tiene como propósito armonizar los procesos contables, establecer los criterios generales que regirán la contabilidad gubernamental y la emisión de información financiera. El ámbito de aplicación de esta ley abarca los tres órdenes de gobierno.

Con el proceso antes descrito, tiene su origen el Consejo Nacional de Armonización Contable (CONAC), constituido como un órgano de coordinación para la 
armonización de la contabilidad gubernamental, tiene por objeto la emisión de las normas contables y lineamientos para la generación de información financiera que aplicarán los entes públicos. Esta normatividad contable es de aplicación general para todos los entes, y se pretende elaborar información financiera armonizada y comparable, es decir, que todos los entes que reciben recursos públicos realicen un registro homogéneo, con las mismas bases y criterios contables, a efecto de facilitar la emisión de información financiera consolidada que permita una mejor interpretación y análisis de la Cuenta Pública, la cual debe permitir una evaluación de las metas y objetivos alcanzados, así como el desempeño de cada ente.

En este contexto, con el propósito de combatir otro aspecto que afecta directamente los resultados de los entes públicos, se establecieron normas para prevenir y detectar la corrupción, por lo tanto, el 18 de julio de 2016, fue aprobada la Ley General del Sistema Nacional Anticorrupción (LGSNA). El estado de Sonora, en cumplimiento a la LGSNA, realizó los trabajos legislativos pertinentes que contribuyeron a la promulgación de la Ley del Sistema Estatal Anticorrupción (LSEA), publicada el 11 de mayo de 2017 en el Boletín Oficial del Estado.

En sus inicios, la auditoría tenía un enfoque de verificar el cumplimiento normativo, lo cual propiciaba que las personas ubicaran a los auditores en un ámbito de detectives, sin embargo, en el sector público, ha evolucionado tomando como referencia la aparición del control interno, por lo tanto, fue necesario establecer normas de supervisión, para posteriormente en los años de 1950 a 1960, enfocarse a la verificación de emitir opinión sobre la razonabilidad de las cifras de los estados financieros. A partir de la década de 1970, ha enfocado su análisis en el cumplimiento de metas y objetivos, para lo cual se desarrollaron normas y procedimientos relacionados con las auditorías al desempeño.

\section{Problema de investigación}

\section{Planteamiento del problema}

Mediante la presente investigación se expone la necesidad de que los entes fiscalizadores del Gobierno de Sonora, impulsen y expongan resultados de trabajos de auditorías al cumplimiento de metas y objetivos, sobre todo demuestren el impacto social para el que fue etiquetado el recurso público a través de los diversos programas y proyectos; por lo que es necesario fomentar la elaboración de las auditorías al desempeño, enfocando sus esfuerzos en brindar la capacitación necesaria para el buen desarrollo de este tipo de revisiones. Esto, promueve la gobernanza económica, eficaz y eficiente y contribuye a la rendición de cuentas hacia la sociedad y a la transparencia a través de dar a conocer cómo, para qué y qué sucede tanto con los recursos del erario, así como los procedimientos administrativos que se llevan a cabo, todo lo anterior, con el propósito de medir los impactos económicos y sociales.

Una vez consultados en la actualidad los portales de internet del entorno gubernamental (ISAF y SECOG), se aprecia la necesidad de reforzar el diseño y elaboración de procedimientos tendientes al desahogo de la rendición de cuentas mediante auditorías al desempeño de los entes fiscalizados; al percibirse nula o escasamente conocidos en el ámbito estatal.

Es pertinente señalar que en la Secretaría de la Contraloría General se han realizado esfuerzos importantes para medir el desempeño de los entes gubernamentales estatales del estado de Sonora, para lo cual diseñaron el Sistema de Control Interno Institucional, mismo que utiliza primordialmente la evaluación mediante indicadores de desempeño y las evaluaciones, diseñando para su operación a los Comités de Control y Desempeño Institucional (COCODI). 


\section{Justificación}

El presente trabajo académico de investigación relacionado con la auditoría al desempeño en el ámbito estatal de Sonora, es un interés que ha surgido por la escasa evidencia documental relacionada con el desarrollo de este tipo de auditorías en el Estado, no obstante, ser un mandato constitucional. Asimismo, las AD son de reciente aplicación en Sonora, debido a que se empezaron a desarrollar en el ejercicio fiscal 2018.

En lo que concierne al ámbito federal, existe más información relacionada con este tipo de auditorías, en especial en la Auditoría Superior de la Federación, así como en la Secretaría de la Función Pública; sin embargo, la difusión de los resultados de las auditorías al desempeño necesita incrementarse, con el objetivo primordial de que las personas encargadas de la administración de recursos públicos en los entes del estado de Sonora, estén conscientes de la relevancia de cumplir las metas y objetivos que por normatividad tienen establecidos. No obstante que en el ámbito gubernamental estatal de Sonora existen esfuerzos enfocados a medir el desempeño institucional, se aprecia la necesidad de reforzar la comunicación de los resultados obtenidos hacia los beneficiarios, en este caso, la ciudadanía.

Es así, que en este trabajo se pretende primeramente identificar la cantidad de auditorías al desempeño realizadas por los entes encargados del control y fiscalización en Sonora, para posteriormente proporcionar un instrumento de apoyo para practicar auditorías al desempeño en las dependencias y entidades, beneficiando directamente a los servidores públicos que desarrollan funciones de auditoría, así como a cualquier persona interesada en el tema que cuente con el perfil de contador público, abogado, administrador de empresa, así como otros perfiles afines.

\section{Objetivos}

\section{Objetivo general}

Evaluar la evolución y relevancia de las auditorías al desempeño en el ámbito estatal con referencia del nivel federal realizadas por el Instituto Superior de Auditoría y Fiscalización (ISAF) y por la Secretaría de la Contraloría General (SECOG) con entes que han sido objeto de fiscalización en el estado de Sonora, para identificar el impacto de beneficio social y económico en la gestión gubernamental estatal.

\section{Objetivos específicos}

a) Exponer la evolución y relevancia de evaluar los resultados de los entes de gobierno respecto a las auditorías al desempeño realizadas por ISAF y SECOG.

b) Proporcionar un instrumento de apoyo para practicar auditorías al desempeño en las dependencias y entidades de la Administración Pública Gubernamental en Sonora que incidan en buenas prácticas.

\section{Planteamiento metodológico}

La investigación versa sobre las auditorías al desempeño realizadas por ISAF y SECOG en el estado de Sonora; para posteriormente proporcionar un instrumento de apoyo para practicar auditorías al desempeño.

\section{Método}

El método utilizado es mixto (deductivo-inductivo), debido a que en primera instancia se aborda el tema de $\mathrm{AD}$ en forma general, retomando antecedentes $\mathrm{y}$ situación actual en México, para después verificar el desarrollo de estas auditorías en el estado de Sonora. 


\section{Enfoque}

La información se analiza desde mixto, primero un aspecto cualitativito, para conocer la situación de la $\mathrm{AD}$ en el estado de Sonora, así como aportar un instrumento de apoyo para su desarrollo, informando a la vez la postura de la propia investigación. El enfoque cuantitativo se requiere para el análisis de cifras de tendencias para observar la evolución a través de los años de este tipo de auditoría.

En lo que concierne al método cualitativo, Bernal (2010, p. 60), tomando como referencia lo expuesto por Bonilla y Rodríguez (2000), señala que "profundiza casos específicos y no a generalizar. Su preocupación no es prioritariamente medir, sino cualificar y describir el fenómeno social a partir de rasgos determinantes, según sean percibidos por los elementos mismos que están dentro de la situación estudiada”.

\section{Tipo de investigación}

Se desarrolla un tipo de investigación documental y descriptiva, donde se analiza e interpreta el contenido de las fuentes consultadas, sean leyes, literatura y artículos académicos, para brindar algunos resultados del origen, conceptos y aplicaciones de la AD.

Al respecto, Bernal (2010, p. 113), retoma un análisis de Salkind (1998), donde se considera como investigación descriptiva aquella en que "se reseñan las características o rasgos de la situación o fenómeno objeto de estudio”.

\section{Alcance}

El alcance es transversal, debido a que el origen de la $\mathrm{AD}$ en México y por ende en Sonora, es a partir del principio de legalidad, por lo tanto, tiene su origen en una Ley, en específico, los artículos 74 y 79 de la CPEUM.

\section{Ámbito geográfico}

Se tiene un ámbito estatal, debido a que los resultados analizados corresponden al estado de Sonora, en específico, se verifican las auditorías al desempeño practicadas en ISAF y SECOG.

\section{Investigación documental}

Los datos se recolectan en forma documental mediante consultas a las bases normativas internacionales y de los tres niveles de gobierno. A la vez de analizar información pública de los portales de internet del ISAF y SECOG; de igual manera, se visitan otras fuentes secundarias entre las que destacan los portales de internet del Congreso de la Unión, Congreso del Estado de Sonora, Supremo Tribunal de Justicia de Sonora, así como visita al sitio www.bidi.uson.mx, donde se analizaron publicaciones académicas de las bases de datos Ebsco Host, Science Direct y Scopus.

Otro sitio visitado corresponde al Portal web de la Secretaría de la Función Pública http://www.funcionpublica.gob.mx/, así como el Portal de la Auditoría Superior de la Federación http://www.asf.gob.mx., entre otras. En el aspecto académico fue visitada la biblioteca del Departamento de Derecho de la Universidad de Sonora, ubicada en Hermosillo, Sonora, con el propósito de reunir bibliografía que nos permitiera brindar un enfoque más amplio y apegado a la situación actual de este tipo de auditorías en el Estado, sin embargo, constatamos que el material es relativamente escaso, ya que únicamente localizamos los libros, Estudio sociológico jurídico sobre el delito de fraude, Normas y Procedimientos de la auditoría integral, Auditoría un enfoque práctico y Auditoría a las Cuentas Públicas. 


\section{Problemas y limitaciones para el desarrollo del tema}

Se ha logrado un trabajo de investigación de los principales aspectos que conforman los pasos a seguir para el desarrollo de auditorías al desempeño en el ámbito gubernamental del estado de Sonora, sin embargo, debe destacarse que la información con carácter pública y al acceso de cualquier persona es relativamente escasa.

Por tal motivo, al momento de querer efectuar un análisis objetivo de los resultados obtenidos en las evaluaciones al desempeño, encontramos que la metodología utilizada no se encuentra debidamente autorizada, es decir, no fue localizada guía metodológica estatal para la elaboración de estas auditorías en el marco normativo del estado de Sonora. Cabe mencionar que en el ámbito federal existen Guías con fundamento legal y procedimientos que conforman el plan de trabajo, el cual hace referencia a las principales etapas de cualquier auditoría, es decir, señalan el objetivo de la revisión, definen su alcance, abordan antecedentes, planeación, ejecución de los trabajos, informe de resultados y su seguimiento.

\section{Marco referencial}

Derivado de la necesidad del hombre de vivir en sociedad, se ha producido una idea de incrementar el patrimonio de las personas. Desde los años cincuenta la doctrina abordaba la importancia que la sociedad brinda al dinero y su acumulación para enriquecerse por parte de las personas, para lo cual se realizaron publicaciones cuyo propósito era dar a conocer como los delitos patrimoniales eran cada vez mayores y recurrentes. Arrollo (1962), en su libro "Estudio sociológico jurídico sobre el delito de fraude” cita lo siguiente:

El medio en que vivimos. Con un poco de exageración podría describirse en estos términos: hoy 1955, parece que la obsesión del hombre en poseer dinero y que esta posesión lo justifica todo; la ebriedad, el divorcio, el adulterio, la homosexualidad, la especulación, el juego, son motivo de comentarios alegres de sociedad que lejos de repudiar a sus autores, los encuentra interesantes. Parece que el bien, el mal, lo justo, lo injusto, no existen para quien escapa de la miseria. En la prisión quedan únicamente los que con anterioridad han caído en ella; los delincuentes que pueden pagar gozan de libertad. El contemporáneo comprende que las costumbres están empapadas de frivolidad; pero nadie trata de arreglarlas, porque apenas tiene tiempo para sobrevivir en la dura lucha económica. (p. 18)

Es importante resaltar que las palabras antes citadas textualmente, fueron plasmadas por su autor en el año 1955, por lo tanto, de allí reviste su importancia, porque al efectuar una comparación de esas ideas contra la realidad actual del año 2019, se infiere inmediatamente que la importancia del dinero continúa siendo la misma, incluso en estas fechas se le brinda mayor interés a lo económico, y en muchas de las veces, la forma de acumular riqueza es contraviniendo las leyes y normas.

En un estudio sobre la evolución de la Ad en Australia, Hossain (2010, p. 20), expone que esta auditoría adquiere su importancia porque está dirigida hacia el rendimiento, la eficiencia, ahorro y productividad de los órganos de la administración pública, y cubre no solo aspectos específicos de la administración, sino también de la actividad de gestión, que incluye organización y gestión sistemas. Por otro lado, McCrae \& Vada (1997, p.203), realizan un estudio donde ponen a discusión la legalidad de las auditorías al desempeño en Australia, las cuales han tenido ese problema desde 1979, aun así, se efectúan revisiones por la Oficina Nacional de Auditoría de Australia. En otro estudio similar en Australia, Funnell \& Wade (2012, p. 434), confirman que la auditoría de desempeño continúa siendo una actividad 
impugnada y su credibilidad en la práctica sigue siendo incierto.

En Brasil, Silveira (2019, p.23), propone una nueva clasificación de los resultados y seguimientos de la auditoría del desempeño, señalando que, durante varias décadas, estas auditorías han ayudado significativamente a mejorar la gestión pública, sin embargo, en el periodo de los años 2004 al 2015, pocos han estudiado el proceso de seguimiento de las situaciones detectadas. Es importante señalar, que el proceso de seguimiento cumplimenta la etapa final de la auditoría, debiendo aclararse cada una de las situaciones con la mayor transparencia posible.

\section{Marco conceptual}

\section{Auditoría}

Primeramente, se define el concepto general de auditoría, ante lo cual se cita lo señalado por De la Peña (2011) "el vocablo auditoría es sinónimo de examinar, verificar, investigar, consultar, revisar, comprobar y obtener evidencias sobre informaciones, registros, procesos, circuitos, etc."

\section{Auditoría integral}

Es importante también abordar la auditoría desde un enfoque integral, es decir, en su totalidad, esto es cuando los trabajos a realizar engloban toda una empresa. Blanco (2004) respecto a la definición de auditoría integral cita el siguiente concepto:

Auditoría integral es el proceso de obtener y evaluar objetivamente, en un periodo determinado, evidencia relativa a la siguiente temática: la información financiera, la estructura del control interno, el cumplimiento de las leyes pertinentes y la conducción ordenada en el logro de las metas y objetivos propuestos; con el propósito de informar sobre el grado de correspondencia entre la temática y los criterios o indicadores establecidos para su evaluación. (p. 16)

\section{Control Interno}

Otro aspecto interesante y primordial en las auditorías es la evaluación del Control Interno, ante lo cual Arens, Randal y Mark (2007), manifiestan la siguiente definición de Control Interno: "proceso diseñado para proporcionar una seguridad razonable con respecto al cumplimiento de los objetivos de la administración en las siguientes categorías: confiabilidad de los informes financieros, eficacia de las operaciones y cumplimiento con las leyes y reglamentos."

\section{Auditoría al desempeño}

La Auditoría Superior de la Federación (ASF) con base en lo establecido por la Organización Internacional de Entidades Fiscalizadoras Superiores (INTOSAI), expone que las auditorías de desempeño "otorgan información, análisis y perspectivas sobre el quehacer gubernamental para minimizar los costos de los recursos empleados; obtener el máximo de los insumos disponibles; lograr los resultados previstos, y verificar el impacto social $y$ económico para la ciudadanía”.

La AD sólo analiza lo que sea decisivo o crítico para el cumplimiento de la misión y de la visión. Se enfoca prácticamente al análisis de los resultados de la entidad, con respecto al logro de los factores críticos de desempeño.

Con base en sus propias normas de auditoría, la Organización Internacional de las Entidades Fiscalizadoras Superiores (INTOSAI) por sus siglas en inglés, define la auditoría del rendimiento, conocida en México como auditoría al desempeño, como "una revisión independiente, objetiva y confiable sobre si las acciones, sistemas, operaciones, programas, actividades u organizaciones del gobierno operan de acuerdo con los principios de economía, eficiencia y eficacia, y sobre si existen áreas de mejora” (p. 2)

Según Raudla, Taro, Agu \& Douglas (2016, p. 219), la auditoría de desempeño es vista como un mecanismo para mejorar la rendición de cuentas en el sector público, 
lo cual tiene semejanza con el enfoque brindado en México. Por su parte Iatco, Ignat, Ungureanu \& Athes (2014, p.253), exponen que la auditoría de desempeño representa un examen y, más particularmente, una evaluación independiente y objetiva del grado en que un programa o una actividad de una entidad pública funciona de manera eficiente, mientras que es productivo. Lo anterior, deja de manifiesto la necesidad de brindar cuentas de los resultados de gestión en el ámbito de gobierno.

La auditoría de desempeño en el sector público representa una evaluación independiente del grado de efectividad y la eficiencia de una determinada actividad, programa o institución, al tiempo que se observan los principios de eficacia (Voiculescu \& Tătaru, 2019, p. 1521). En esta investigación se observa que los autores relacionan la auditoria al desempeño con la eficacia y efectividad.

\section{Marco legal}

Las auditorías gubernamentales son de varios tipos y dependen del enfoque que se requiera, así como de los objetivos de la auditoría, dentro de estas auditorías se encuentra la enfocada al desempeño, por lo tanto, todas se rigen por una serie de leyes y reglamentos los cuales emanan de la Constitución Política de los Estados Unidos Mexicanos.
En la presente investigación se abordará el Marco Jurídico más idóneo, es decir, el primordial para realizar una auditoría, toda vez que las leyes y procedimientos para tal fin son una cantidad considerable, no obstante, es importante mencionar que en el ámbito gubernamental ya sea federal, estatal o municipal, el actuar de los auditores se debe basar en letra escrita, y lo más importante, la legislada. Además de hacer cumplir de la misma forma lo legislado que no siempre significa lo más eficiente.

A continuación, se presenta resumen de las principales normas jurídicas internacionales y en los tres ámbitos de gobierno: ISSAI 300. Principios Fundamentales de la Auditoría de Desempeño.

Esta norma persigue establecer una base común de conocimiento sobre la naturaleza de la Auditoría de Desempeño, incluyendo los principios que deben aplicarse antes y durante las principales etapas del proceso de auditoría. Las Directrices Generales de Auditoría sobre auditoría de desempeño (ISSAIs 30003099) y auditoría de cumplimiento (ISSAIs 4000-4999), facilitan directrices adicionales al auditor. (ISSAIs 10004999) contienen los requerimientos recomendados sobre auditoría financiera. 


\section{Marco jurídico a nivel Federal}

Tabla 1. Marco jurídico federal

\begin{tabular}{|l|l|l|}
\hline \multicolumn{1}{|c|}{ Ley } & \multicolumn{1}{|c|}{ Objeto } & \multicolumn{1}{|c|}{ Artículos } \\
\hline $\begin{array}{l}\text { Constitución Política de los Estados } \\
\text { Unidos Mexicanos }\end{array}$ & $\begin{array}{l}\text { Se establecen los derechos y obligaciones } \\
\text { esenciales de los ciudadanos y gobernantes }\end{array}$ & $\begin{array}{l}\text { 31, 74, 79 Fr I } \\
\text { y 134 }\end{array}$ \\
\hline $\begin{array}{l}\text { Ley de Fiscalización y Rendición de } \\
\text { Cuentas de la Federación }\end{array}$ & $\begin{array}{l}\text { Evaluar los resultados de la gestión financiera } \\
\text { de las entidades fiscalizadas; comprobar si se } \\
\text { observó lo dispuesto en el Presupuesto }\end{array}$ & $\begin{array}{l}\text { Reglamentar } \\
\text { los artículos 74, } \\
\text { fracciones II y } \\
\text { VI, y } \\
\text { CPEUM }\end{array}$ \\
\hline $\begin{array}{l}\text { Ley Orgánica de la Administración } \\
\text { Pública Federal }\end{array}$ & $\begin{array}{l}\text { Establece las bases de organización de la lán } \\
\text { Administración Pública Federal, centralizada y } \\
\text { paraestatal }\end{array}$ & $\begin{array}{l}\text { y Vr I, III, IV } \\
\text { y V }\end{array}$ \\
\hline
\end{tabular}

Fuente: Elaboración propia con análisis de la CPEUM.

\section{Sistema de Evaluación del Desempeño}

Es importante destacar que, desde el 31 de marzo de 2008, en la Auditoría Superior de la Federación, existe el Sistema de Evaluación del Desempeño (SED), el cual tiene su origen en el artículo 2 fracción LI de la Ley Federal de Presupuesto y Responsabilidad Hacendaria. Es aquí donde se define a este Sistema de la siguiente forma:

el conjunto de elementos metodológicos que permiten realizar una valoración objetiva del desempeño de los programas, bajo los principios de verificación del grado de cumplimiento de metas y objetivos, con base en indicadores estratégicos y de gestión que permitan conocer el impacto social de los programas y de los proyectos.
El objetivo primordial del SED es mejorar la función pública, lo que significa prestar mejores servicios, eficientar el uso del presupuesto, mejorar el ambiente de trabajo y desarrollar en los funcionarios sus capacidades de liderazgo y de innovación. El SED comprende el estudio y análisis de: objetivos y metas de las dependencias, entidades o programas, análisis del control interno, reglas de operación de cada programa, padrón de beneficiados del programa, determinación de indicadores y elaboración de la matriz de indicadores, avances del programa y de las actividades, elaboración de la matriz de riesgos, estudio de Manuales de organización, estudio de Manuales de procedimientos y resultados de evaluaciones anteriores. 


\section{Marco jurídico a nivel Estatal}

Tabla 2. Marco jurídico estatal

\begin{tabular}{|l|l|c|}
\hline \multicolumn{1}{|c|}{ Ley } & \multicolumn{1}{|c|}{ Objeto } & Artículos \\
\hline $\begin{array}{l}\text { Constitución Política del Estado de } \\
\text { Sonora }\end{array}$ & $\begin{array}{l}\text { Se establecen los derechos y obligaciones } \\
\text { esenciales de los ciudadanos y gobernantes }\end{array}$ & 67 \\
\hline $\begin{array}{l}\text { Ley Orgánica del Poder Ejecutivo } \\
\text { del Estado de Sonora }\end{array}$ & $\begin{array}{l}\text { Establece la estructura, funcionamiento y bases } \\
\text { de organización del Poder Ejecutivo del Estado } \\
\text { de Sonora }\end{array}$ & $\begin{array}{c}\text { 26 inciso c, Fr III, inciso } \\
\text { E Fr I y II }\end{array}$ \\
\hline $\begin{array}{l}\text { Ley de Fiscalización Superior para el } \\
\text { Estado de Sonora }\end{array}$ & $\begin{array}{l}\text { Regular la revisión, auditoria y fiscalización } \\
\text { superior de los estados financieros y cuentas } \\
\text { públicas estatal y municipales }\end{array}$ & 7 \\
\hline
\end{tabular}

Fuente: Elaboración propia con análisis de la Constitución Política de Sonora.

\section{Marco jurídico a nivel Municipal}

Tabla 3. Marco jurídico municipal

\begin{tabular}{|c|l|c|}
\hline \multicolumn{1}{|c|}{ Ley } & \multicolumn{1}{|c|}{ Objeto } & Artículos \\
\hline $\begin{array}{l}\text { Ley de gobierno y administración } \\
\text { municipal }\end{array}$ & $\begin{array}{l}\text { Regular las bases para la integración y } \\
\text { organización del territorio, la población, el } \\
\text { gobierno y la administración pública municipal }\end{array}$ & 61,94 y 95 \\
\hline
\end{tabular}

Fuente: Elaboración propia con análisis de la Ley de gobierno y administración municipal.

\section{Entes facultados para efectuar auditorías}

Los Entes fiscalizadores en México y Sonora son:

Ámbito federal

a) Auditoría Superior de la Federación

b) Secretaría de la Función Pública

Ámbito estatal

c) Instituto Superior de Auditoría y Fiscalización del Estado de Sonora d) Secretaría de la Contraloría General

e) Contraloría Municipal (Órgano de Control y Evaluación Gubernamental)

\section{Auditoría Superior de la Federación (Ámbito federal)}

La revisión de la Cuenta Pública está a cargo de la Cámara de Diputados, la cual se apoya para tal efecto en la Auditoría Superior de la Federación (ASF), misma que tiene a su cargo la fiscalización de dicha Cuenta. 


\section{Secretaría de la Función Pública (Ámbito federal)}

La Secretaría de la Función Pública, dependencia del Poder Ejecutivo Federal, vigila que los servidores públicos federales se apeguen a la legalidad durante el ejercicio de sus funciones, sanciona a los que no lo hacen así; promueve el cumplimiento de los procesos de control y fiscalización del gobierno federal, de disposiciones legales en diversas materias, dirige y determina la política de compras públicas de la Federación, coordina y realiza auditorías sobre el gasto de recursos federales, coordina procesos de desarrollo administrativo, gobierno digital, opera y encabeza el Servicio Profesional de Carrera, coordina la labor de los órganos internos de control en cada dependencia del gobierno federal y evalúa la gestión de las entidades, también a nivel federal.

\section{Instituto Superior de Auditoría y Fiscalización del Estado de Sonora (Ámbito estatal)}

El Instituto Superior de Auditoría y Fiscalización del Estado de Sonora, es un órgano constituido con autonomía presupuestaria, técnica y de gestión para establecer su organización interna, funcionamiento y resoluciones; con las facultades legales como Órgano de Fiscalización Superior del Congreso del Estado para realizar la auditoría de los Estados Financieros, la revisión y fiscalización de la Cuenta Pública del Estado y de los Municipios y demás sujetos fiscalizados, así como las demás actividades necesarias para el eficaz cumplimiento de sus funciones y competencia de acuerdo con lo establecido en la Constitución Política del Estado Libre y Soberano de Sonora, la Ley de Fiscalización Superior para el Estado de Sonora y el Reglamento, Interior del ISAF así como las demás disposiciones legales aplicables.

\section{Secretaría de la Contraloría General. (Ámbito estatal)}

La Secretaría de la Contraloría General es una dependencia de la administración pública directa, conformada con base en la Ley Orgánica del Poder ejecutivo del Estado de Sonora, la cual tiene facultades en materia de control, desarrollo administrativo, control gubernamental, participación social y vinculación, así como en transparencia y combate a la corrupción.

Objetivos Estratégicos

- Establecer los mecanismos de control y evaluación gubernamental que aseguren el cumplimiento del marco normativo.

- Promover acciones en Dependencias y Entidades para mejorar su desempeño y calidad en sus servicios.

- Fomentar la participación ciudadana en la gestión de gobierno y acordar acciones con los sectores público, social y privado para promover una cultura de transparencia y combate a la corrupción.

\section{Contraloría Municipal - (Ámbito municipal)}

La contraloría municipal tiene su base de creación en la Ley de Gobierno y Administración Municipal, misma que hace referencia a que el Ayuntamiento deberá contar con un Sistema Administrativo Interno de Control y Evaluación Gubernamental, cuya finalidad consiste en controlar la legalidad, honradez, lealtad, imparcialidad y eficiencia en el servicio público. 


\section{Resultados y discusión}

Los resultados de las Auditorías al Desempeño han sido destacados en los últimos años, lo cual ha permitido una mejor revisión de la gestión pública de los entes, permitiendo así detectar áreas de oportunidad que permiten alinear el impacto económico y social de la operación pública. Por lo tanto, la Auditoría Superior de la Federación, emprendió acciones para disminuir la brecha entre las auditorías financieras y de desempeño.

En México, la ASF desde su creación, ha realizado un total de 17,631 auditorías, de las cuales, con enfoque de desempeño son 2,385 , lo que representa un $13.5 \%$ con respecto al total de auditorías. Sin embargo, debido a la necesidad de rendición de cuentas a la sociedad y, siendo esta herramienta un aspecto fundamental para tal fin, para la Cuenta Pública 2017, el porcentaje de auditorías al desempeño se incrementó considerablemente hasta alcanzar un $42.9 \%$ de las auditorías ejecutadas, es decir, por cada 10 auditorías realizadas 4 son con enfoque de desempeño. Debido a que se ejecutaron 1,682 auditorías y 722 son de enfoque de desempeño.

Gráfico 1. Auditorías realizadas por ASF periodos 2000-2017 y Cuenta Pública 2017

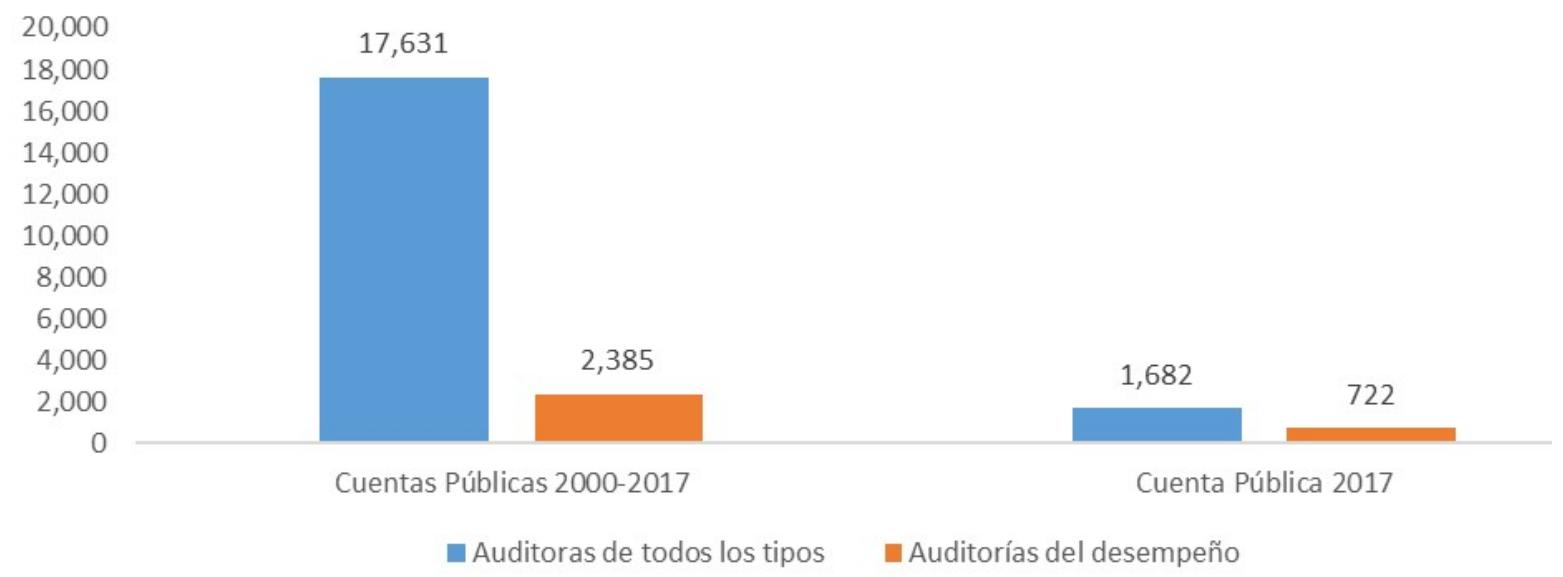

Fuente: elaboración propia con cifras del portal http://www.asfdatos.gob.mx/

El periodo contemplado en la presente investigación es a partir del ejercicio 2018, debido a que en este año es cuando inician las auditorías al desempeño en Sonora. Derivado de lo anterior, al consultar los portales de internet del ISAF y SECOG, se constató que se ejecutaron 148 auditorías con enfoque de desempeño, 112 elaboradas por ISAF y 36 por SECOG.

La investigación realizada es relevante porque es una situación novedosa, debido a que las auditorías al desempeño en el estado de Sonora, solamente se han realizado a partir del ejercicio fiscal 2018; no obstante, la encomienda constitucional de realizarlas proviene desde el 31 de marzo de 2008, al publicarse en el Diario Oficial de la Federación, el Acuerdo por el que se establecen las disposiciones generales del Sistema de Evaluación del Desempeño. Para que este tipo de auditorías fueran contempladas en los programas de trabajo del ISAF y SECOG, trascurrieron aproximadamente 10 años.

A continuación, se muestra el resultado de la consulta realizada a los portales de internet de ISAF y SECOG, cuyo propósito fue identificar la cantidad de auditorías al desempeño realizadas en el ejercicio fiscal 2018. 


\section{Auditorías al desempeño en ISAF}

Las auditorías al desempeño en ISAF, se implementaron por primera vez en la revisión de la Cuenta Pública 2017; debido a que anteriormente no se ejecutaban este tipo de auditorías en Sonora. En esta Cuenta Pública, el ISAF ejecutó un total de 672 auditorías de todos los tipos, de las cuales 112 son con enfoque de desempeño, lo cual equivale al $17 \%$ del total; por lo tanto, por cada 10 auditorías realizadas 2 corresponden al rubro de desempeño. A continuación, se presenta tabla y gráfica de los resultados.

Tabla 4. Auditorías al desempeño ejecutadas en Cuenta Pública 2017 (ISAF)

\begin{tabular}{|l|c|}
\hline \multicolumn{1}{|c|}{ Entes fiscalizados } & Número de auditorías del desempeño \\
\hline Dependencias & 14 \\
\hline Entidades, fondos y fideicomisos & 69 \\
\hline Organismos autónomos & 8 \\
\hline Órganos desconcentrados & 12 \\
\hline Poder Judicial & 1 \\
\hline Poder legislativo & 1 \\
\hline Fondos federales & 7 \\
\hline Total auditorías al desempeño & 112 \\
\hline Total de auditorías de todos los tipos & 672 \\
\hline \% Auditorías al desempeño & $17 \%$ \\
\hline
\end{tabular}

Fuente: Cuenta Pública del estado de Sonora 2017.

\section{Auditorías al desempeño en SECOG}

Las auditorías al desempeño en SECOG, también fueron implementaron en el año 2018, efectuando 36 auditorías de este tipo, todas fueron realizadas en
Entidades del sector ejecutivo; por lo tanto, en el ámbito de las Dependencias no se realizaron auditorías al desempeño. A continuación, se presenta una tabla de las entidades en las cuales se realizaron estas revisiones. 
Tabla 5. Programa anual de auditorías al desempeño 2018 (SECOG)

\begin{tabular}{|c|c|c|c|c|c|}
\hline Entidad & $\begin{array}{l}\text { Auditorías } \\
\text { al } \\
\text { desempeño }\end{array}$ & Entidad & $\begin{array}{l}\text { Auditorías } \\
\text { al } \\
\text { desempeño }\end{array}$ & Entidad & $\begin{array}{l}\text { Auditorías } \\
\text { al } \\
\text { desempeño }\end{array}$ \\
\hline CECC & 1 & FIDESON & 1 & JCES & 1 \\
\hline CEA/FOSSI & 2 & ICATSON & 1 & RADIO SONORA & 1 \\
\hline CECOP & 1 & ICES & 1 & SEES & 1 \\
\hline CECYTES & 1 & CRESON & 1 & $\begin{array}{l}\text { SEGURO } \\
\text { POPULAR }\end{array}$ & 1 \\
\hline CEDES/PROAES & 2 & IMPULSOR & 1 & SSS & 1 \\
\hline CEDIS & 1 & ISC & 1 & TELEMAX & 1 \\
\hline СOBACH & 1 & ISEA & 1 & UES & 1 \\
\hline CODESON & 1 & ISIE & 1 & UNISIERRA & 1 \\
\hline COFETUR/APISON & 1 & ISJ & 1 & UTH & 1 \\
\hline CONALEP & 1 & ISM & 1 & UTPP & 1 \\
\hline COVES & 1 & ISSPE & 1 & & \\
\hline DIF SONORA & 1 & ISSSTESON & 1 & & \\
\hline
\end{tabular}

Fuente: Agenda estratégica 2018: Programa Anual de Auditorías (SECOG).

En la tabla se aprecia que en la mayoría de las entidades del poder ejecutivo se realizó una auditoría al desempeño, a excepción de la Comisión Estatal del Agua (CEA/FOSSI) y la Comisión de Ecología y Desarrollo Sustentable del Estado de Sonora (CEDES/PROAES), en las cuales se realizaron dos auditorías de este tipo.

\section{Programa de trabajo para una auditoría al desempeño}

Al final de este trabajo se muestra un anexo, mismo que detalla en orden cronológico las principales actividades para el desahogo de una auditoría al 
desempeño; la cual fue elaborada por el Secretario de la Contraloría y Modernización Administrativa del gobierno del Estado de Durango, procediendo por nuestra parte a una breve modificación y adecuación a las necesidades del presente trabajo.

\section{Conclusión}

Es pertinente aclarar la importancia de medir el desempeño de los entes y programas gubernamentales en el Estado de Sonora. "Si no se mide lo que se hace, no se puede controlar y si no se puede controlar, no se puede dirigir y si no se puede dirigir no se puede mejorar" (Peter Druker).

Al momento de una auditoría al desempeño, es preciso verificar que la entidad cumpla con la norma legal, sin dejar de lado la percepción que tienen los ciudadanos respecto a dicha entidad. Este tipo de auditorías al desempeño, facilitan identificar las causas o áreas de oportunidad que limitan la consecución de los objetivos, proponiendo medidas preventivas y correctivas que apoyen a la dependencia o entidad al logro de sus fines, así como aprovechar mejor los recursos que tiene asignados y que el otorgamiento de sus servicios sea oportuno, confiable y completo.

Del análisis al portal de internet de la Auditoría Superior de la Federación, se constató que, en Sonora, ha ejecutado 383 auditorías de todos los tipos, sin embargo, solamente 22 corresponden al rubro desempeño, lo que equivale al $5.7 \%$, cifra muy pequeña con respecto a los otros tipos de auditorías.

Sin embargo, a nivel México, debido a la necesidad de rendición de cuentas a la sociedad y, siendo esta herramienta un aspecto fundamental para tal fin, para la Cuenta Pública 2017, el porcentaje de auditorías al desempeño se incrementó considerablemente hasta alcanzar un $42.9 \%$ de las auditorías ejecutadas, es decir, por cada 10 auditorías realizadas 4 son con enfoque de desempeño, debido a que se ejecutaron 1,682 auditorías y 722 son de enfoque de desempeño.

Por su parte, los Órganos facultados para realizar auditorías en Sonora, empezaron a realizar auditorías al desempeño en el ejercicio fiscal 2018. El Instituto Superior de Auditoría y Fiscalización (ISAF) realizó 112 auditorías al desempeño y la Secretaría de la Contraloría General (SECOG) realizó 36 en el mismo periodo.

Cabe señalar que la encomienda de realizar auditorías al desempeño inicia el 31 de marzo de 2018, al aprobarse el Sistema de Evaluación del Desempeño (SED), no obstante, transcurrieron 10 años, para que, en Sonora, dieran inicio las auditorías con carácter de desempeño.

Se proporciona un instrumento de apoyo para practicar auditorías al desempeño en las dependencias y entidades de la Administración Pública Gubernamental en el ámbito estatal de Sonora, el cual es de carácter enunciativo, dirigido a los servidores públicos y cualquier persona interesada con perfil de auditoría y contabilidad.

Por último, se considera importante mencionar que esta modalidad de auditoría debe ser enriquecida en forma permanente, para ser aplicada en todos los ámbitos y niveles de gobierno. Es por ello que las autoridades competentes en todos los ámbitos de gobierno, deberían emprender acciones tendientes primeramente a brindar capacitación a todos los servidores públicos que realizan auditorías, para enfocar esfuerzos en la práctica de las mismas. 
Anexo 1. Programa de trabajo de una auditoría al desempeño

Una vez expuestas las necesidades de cada una de las etapas de la planeación general, inicio de auditoría, planeación detallada, criterios de auditoría y ejecución de la auditoría, se muestra un orden cronológico de las principales actividades para el desahogo de la revisión.

Tabla 6. Programa de trabajo de una auditoría al desempeño

\begin{tabular}{|c|c|c|}
\hline & Persona Responsable & Actividades \\
\hline & \multicolumn{2}{|l|}{ Inicio del procedimiento } \\
\hline 1 & $\begin{array}{l}\text { El Titular del Órgano de Control y } \\
\text { Desarrollo Administrativo (OIC), } \\
\text { de conformidad con sus } \\
\text { atribuciones. }\end{array}$ & $\begin{array}{l}\text { Decide la realización de auditoría, con base en el Programa Anual de } \\
\text { Trabajo aprobado, por petición de la parte interesada, por queja, } \\
\text { denuncia o por solicitud de autoridad superior competente. }\end{array}$ \\
\hline 2 & $\begin{array}{l}\text { El Titular del OIC o Supervisor de } \\
\text { auditoría, de conformidad con sus } \\
\text { atribuciones. }\end{array}$ & $\begin{array}{l}\text { Analiza perfiles y disponibilidad de personal, designa el equipo de } \\
\text { auditores e instruye se elabore el proyecto de programa específico de } \\
\text { auditoría y cronograma de actividades, carta de planeación, orden de } \\
\text { auditoría y requerimiento inicial de información. }\end{array}$ \\
\hline 3 & Supervisor de auditoría & $\begin{array}{l}\text { Recibe y revisa proyecto de programa específico de auditoría y } \\
\text { cronograma de actividades, carta de planeación, orden de auditoría y } \\
\text { requerimiento inicial de información, y en su caso, corrige. }\end{array}$ \\
\hline 4 & El Titular del OIC & $\begin{array}{l}\text { Firma la Orden de Auditoría y con base en disponibilidad, autoriza o } \\
\text { gestiona la asignación de los recursos (vehículos, gasolina, viáticos, } \\
\text { computadoras portátiles, etc.) al equipo de auditores. }\end{array}$ \\
\hline 5 & Supervisor y Auditores & $\begin{array}{l}\text { Se constituye en las oficinas del Titular del área auditada, entrega } \\
\text { orden de auditoría y el requerimiento inicial de información, presenta } \\
\text { al equipo de auditores y levanta acta de Inició de Auditoría. } \\
\text { Entrega copia de la orden de auditoría al Titular del OIC o al } \\
\text { Supervisor de auditoría, según corresponda. }\end{array}$ \\
\hline
\end{tabular}


Tabla 6. Continuación...

\begin{tabular}{|c|c|c|}
\hline & Persona Responsable & Actividades \\
\hline & Inicio del procedimiento & \\
\hline 6 & $\begin{array}{l}\text { Área Auditada de la Dependencia } \\
\text { o Entidad u Organismo Auxiliar }\end{array}$ & $\begin{array}{l}\text { Recibe orden de auditoría, sella el acuse y gira instrucciones al } \\
\text { personal a su cargo para facilitar el trabajo de auditoría. }\end{array}$ \\
\hline 7 & Supervisor de auditoría & $\begin{array}{l}\text { Registra en el sistema correspondiente el inicio de auditoría. La fecha } \\
\text { de alta debe coincidir con la fecha de emisión de la orden de } \\
\text { auditoría, teniendo tres días hábiles para notificarlo. En caso de que la } \\
\text { acción se inicie con desfase a lo programado, se deberá justificar la } \\
\text { causa del mismo. }\end{array}$ \\
\hline \multirow[t]{4}{*}{8} & Auditor & $\begin{array}{l}\text { Lleva a cabo el procedimiento de auditoría al desempeño conforme al } \\
\text { programa específico aprobado y registra su avance en el cronograma } \\
\text { de actividades, para ello: }\end{array}$ \\
\hline & & $\begin{array}{l}\text { Elabora y aplica en coordinación con el jefe inmediato el instrumento } \\
\text { para el estudio y evaluación del control interno. }\end{array}$ \\
\hline & & $\begin{array}{l}\text { Modifica en su caso, conforme al resultado del estudio y evaluación } \\
\text { del control interno, el programa específico de auditoría, previa } \\
\text { consulta con su Supervisor. }\end{array}$ \\
\hline & & $\begin{array}{l}\text { Selecciona en su caso, la muestra y deja evidencia de los criterios de } \\
\text { selección aplicados en los papeles de trabajo. }\end{array}$ \\
\hline \multirow[t]{3}{*}{9} & Auditor & $\begin{array}{l}\text { Analiza la información recibida y elabora cédulas sumarias, analíticas } \\
\text { y subanalíticas que en su caso requieran. El análisis de la información } \\
\text { lo realiza conforme a lo siguiente: }\end{array}$ \\
\hline & & Realiza estudio general y conclusiones preliminares \\
\hline & & $\begin{array}{l}\text { Identifica del Ente auditado su marco jurídico, operación, estructura, } \\
\text { recursos y sistemas de planeación e información. }\end{array}$ \\
\hline
\end{tabular}


Tabla 6. Continuación...

\begin{tabular}{|c|c|}
\hline Persona Responsable & Actividades \\
\hline Inicio del procedimiento & \\
\hline & $\begin{array}{l}\text { Realiza una evaluación preliminar sobre los diversos aspectos } \\
\text { relacionados con el cumplimiento de su misión, visión, valores y } \\
\text { objetivos. }\end{array}$ \\
\hline & $\begin{array}{l}\text { Identifica fortalezas, oportunidades, debilidades y amenazas que } \\
\text { enfrenta el Ente auditado y determina en forma preliminar los } \\
\text { factores críticos de desempeño y los indicadores de desempeño } \\
\text { correlativos. }\end{array}$ \\
\hline & Realiza planeación de la auditoría. \\
\hline & $\begin{array}{l}\text { Determina el marco jurídico precisando la naturaleza jurídica del } \\
\text { Ente auditado, en el sentido de si se trata de una entidad, dependencia } \\
\text { o fideicomiso de inversión o de administración, etc., y las distintas } \\
\text { leyes y otras disposiciones legales que afecten específicamente al } \\
\text { Ente. }\end{array}$ \\
\hline & $\begin{array}{l}\text { Identifica el propósito del Ente, ya sea que esté formalmente } \\
\text { manifiesto en declaraciones específicas, o que sea necesario derivarlo } \\
\text { de los documentos fundamentales mencionados en la actividad } \\
\text { anterior. }\end{array}$ \\
\hline & $\begin{array}{l}\text { Analiza la operación del Ente para el diseño posterior de las pruebas } \\
\text { y particularmente para interpretar los resultados de esas pruebas. }\end{array}$ \\
\hline & $\begin{array}{l}\text { Plasma de manera sencilla la operación del Ente a través de un } \\
\text { diagrama, mapa o modelo descriptivo (de preferencia acompañado de } \\
\text { un diagrama de procesos) con la finalidad de entender fácilmente los } \\
\text { aspectos fundamentales de la operación. }\end{array}$ \\
\hline
\end{tabular}


Tabla 6. Continuación...

\begin{tabular}{|c|c|}
\hline Persona Responsable & Actividades \\
\hline Inicio del procedimiento & \\
\hline & $\begin{array}{l}\text { Analiza la estructura de operación del Ente Auditado e identifica si } \\
\text { cuenta con un esquema de Gobierno Corporativo, como puede ser un } \\
\text { Junta Directiva, Junta de Gobierno o cualquier otro órgano directivo, } \\
\text { que tome decisiones y que vigile las acciones de la dirección general. }\end{array}$ \\
\hline & $\begin{array}{l}\text { Identifica los principales recursos (tangibles e intangibles) que } \\
\text { maneja el Ente, tanto los humanos como los materiales, financieros y } \\
\text { tecnológicos, los ubica dentro de la estructura de la operación y } \\
\text { evalúa la eficacia y eficiencia con la que están siendo utilizados. }\end{array}$ \\
\hline & $\begin{array}{l}\text { Conoce a profundidad el sistema de información financiera y de } \\
\text { operación del Ente, es decir, cómo se mide el desempeño por área, } \\
\text { actividad o proceso, y en su conjunto, cómo y a quien le reportan los } \\
\text { resultados de su desempeño; como informa sobre el grado de } \\
\text { cumplimiento con su misión, visión y objetivos. }\end{array}$ \\
\hline & $\begin{array}{l}\text { Determina los factores críticos de desempeño del Ente, ya que, a } \\
\text { través de verificar su grado de logro, evalúa el cumplimiento de los } \\
\text { objetivos estratégicos y de la misión y visión. }\end{array}$ \\
\hline & $\begin{array}{l}\text { Verifica que el Ente cuente con un adecuado sistema de control y } \\
\text { administración de riesgos, formalmente establecido y que funcione, } \\
\text { que le permita confrontar exitosamente las amenazas. }\end{array}$ \\
\hline & $\begin{array}{l}\text { Realiza la evaluación preliminar del desempeño del Ente, siendo ésta } \\
\text { la base para la formulación del diagnóstico inicial y la planeación y } \\
\text { ejecución del resto del trabajo de auditoría. }\end{array}$ \\
\hline & $\begin{array}{l}\text { Determina el alcance general que se dará a la evaluación; es decir, } \\
\text { cuáles son las áreas, proyectos, funciones, procesos o actividades del } \\
\text { Ente Auditado que serán objeto de la evaluación del desempeño. }\end{array}$ \\
\hline
\end{tabular}


Tabla 6. Continuación...

\begin{tabular}{|c|c|}
\hline Persona Responsable & Actividades \\
\hline Inicio del procedimiento & \\
\hline & Identifica áreas críticas que serán prioritarias u oportunas evaluarlas. \\
\hline & $\begin{array}{l}\text { Realiza una evaluación al control interno que apoya el logro de } \\
\text { objetivos y la obtención de resultados, así como específicamente el } \\
\text { aplicable a la documentación, información, control y registro de los } \\
\text { datos que alimentan a los indicadores de desempeño. }\end{array}$ \\
\hline & $\begin{array}{l}\text { Define los criterios de evaluación que se aplicarán en la fase de } \\
\text { ejecución, mismos que son la base de comparación que utilizará el } \\
\text { auditor en su tarea de opinar sobre el cumplimiento de objetivos } \\
\text { estratégicos y obtención de resultados. }\end{array}$ \\
\hline & Verifica la evaluación de los indicadores para una mejora continua. \\
\hline & $\begin{array}{l}\text { Formula el programa de trabajo suficientemente detallado, preciso y } \\
\text { con procedimientos de auditoría específicamente diseñados para el } \\
\text { Ente y cada una de sus áreas sujetas a revisión, para que la auditoría } \\
\text { se realice con eficacia, eficiencia y orden. }\end{array}$ \\
\hline & $\begin{array}{l}\text { Ejecuta los procedimientos de análisis y auditoría señalados en el } \\
\text { Programa de Trabajo, aplicando los criterios de evaluación } \\
\text { mencionados anteriormente. }\end{array}$ \\
\hline & $\begin{array}{l}\text { Formula un modelo o diagrama descriptivo de cada una de las áreas } \\
\text { objeto de la evaluación, que le permita al equipo de auditoría tener la } \\
\text { visión de conjunto sobre lo que está evaluando. }\end{array}$ \\
\hline & $\begin{array}{l}\text { Efectúa un estudio analítico de la ejecución del presupuesto, } \\
\text { complementario al realizado en la fase de estudio general y } \\
\text { conclusiones preliminares. }\end{array}$ \\
\hline
\end{tabular}


Tabla 6. Continuación...

\begin{tabular}{|c|c|c|}
\hline & Persona Responsable & Actividades \\
\hline & Inicio del procedimiento & \\
\hline & & $\begin{array}{l}\text { Verifica cifras de indicadores, hace pruebas sobre las fuentes de } \\
\text { información, compara contra metas, investiga las variaciones y } \\
\text { revalúa sí los indicadores son suficientes e idóneos para medir el } \\
\text { desempeño. }\end{array}$ \\
\hline & & $\begin{array}{l}\text { Analiza los informes y reportes preparados por el Ente en relación } \\
\text { con el logro de los objetivos y metas, indicadores aplicados y } \\
\text { resultados obtenidos. }\end{array}$ \\
\hline & & $\begin{array}{l}\text { Determina el grado de logro de cada uno de los factores críticos de } \\
\text { desempeño, con base en indicadores y considerando otras actividades } \\
\text { y acciones realizadas en el curso de la auditoría. }\end{array}$ \\
\hline & & $\begin{array}{l}\text { Investiga si se han materializado riesgos que pudieran haber afectado } \\
\text { la consecución de objetivos estratégicos y metas, evaluando el grado } \\
\text { de control y administración sobre los mismos. }\end{array}$ \\
\hline & & $\begin{array}{l}\text { Analiza si se están produciendo o realizando bienes, servicios y } \\
\text { actividades no necesarios para el logro de los objetivos estratégicos, o } \\
\text { por lo contrario, si existe carencia de ellos o si su calidad es } \\
\text { insatisfactoria. }\end{array}$ \\
\hline & & $\begin{array}{l}\text { Verifica el clima laboral dentro del Ente, el comportamiento de } \\
\text { seguridad e higiene, como factores que pueden afectar el desempeño. }\end{array}$ \\
\hline & & $\begin{array}{l}\text { Formula la evaluación final sobre el desempeño del Ente, } \\
\text { identificando los elementos clave para fundamentar la evaluación. }\end{array}$ \\
\hline 10 & Supervisor de auditoría & $\begin{array}{l}\text { Supervisa los trabajos de auditoría dejando constancia de su } \\
\text { actuación mediante su firma en los papeles de trabajo y en su caso, } \\
\text { determina nuevos alcances o elementos a aplicar. }\end{array}$ \\
\hline
\end{tabular}


Tabla 6. Continuación...

\begin{tabular}{|c|c|c|}
\hline & Persona Responsable & Actividades \\
\hline & Inicio del procedimiento & \\
\hline 11 & $\begin{array}{l}\text { Área auditada de la Dependencia } \\
\text { o Entidad }\end{array}$ & $\begin{array}{l}\text { Brinda apoyo y proporciona información solicitada por los auditores: } \\
\text { - Da acceso a las áreas. } \\
\text { - Prepara información preferentemente en original, en su caso, integra } \\
\text { copias y entrega a los auditores, quienes devolverán los originales al } \\
\text { concluir la revisión. } \\
\text { - Contesta y firma cuestionarios, atiende entrevistas y da respuesta a } \\
\text { los requerimientos de información. } \\
\text { - Cuando se le solicita debe entregar documentación certificada. } \\
\text { - Durante la ejecución de los trabajos de auditoría establece constante } \\
\text { comunicación con los auditores para facilitar el proceso. }\end{array}$ \\
\hline 12 & Auditor & $\begin{array}{l}\text { Revisa que las respuestas y documentación recibida sean acordes a lo } \\
\text { solicitado, en su caso, aclara inmediatamente con el área auditada. }\end{array}$ \\
\hline 13 & Auditor & $\begin{array}{l}\text { Analiza y clasifica la información a través de la aplicación de } \\
\text { técnicas de auditoría, para que coordinadamente con el supervisor } \\
\text { proyecten las posibles observaciones. }\end{array}$ \\
\hline 14 & Auditor & $\begin{array}{l}\text { Llena cédulas de observaciones en cualquier momento de la } \\
\text { ejecución de la auditoría, y pueden ser: observaciones con presunta } \\
\text { responsabilidad administrativa resarcitoria, disciplinaria y } \\
\text { observaciones de control interno e implementación de acciones de } \\
\text { mejora. }\end{array}$ \\
\hline 15 & Supervisor de auditoría & $\begin{array}{l}\text { Analiza las observaciones determinadas y verifica se cuente con la } \\
\text { evidencia suficiente, competente, relevante y útil que las soporte, } \\
\text { antes de ser entregadas al Ente. Valora su procedencia y deja } \\
\text { constancia con su firma de la supervisión efectuada en la cédula } \\
\text { correspondiente. }\end{array}$ \\
\hline
\end{tabular}


Tabla 6. Continuación...

\begin{tabular}{|c|c|c|}
\hline & Persona Responsable & Actividades \\
\hline & Inicio del procedimiento & \\
\hline 16 & Titular del OIC & $\begin{array}{l}\text { Remite al Titular del área de Responsabilidades del ÓIC o en su caso } \\
\text { a la Coordinación General de Substanciación y Responsabilidades, el } \\
\text { Informe de Presunta Responsabilidad Administrativa, mediante } \\
\text { Oficio, obteniendo acuse respectivo. Se podrán remitir antes del } \\
\text { cierre de la auditoría, sin consulta jurídica y sin hacerlo de } \\
\text { conocimiento del Ente, la evidencia certificada de las observaciones } \\
\text { con presunta responsabilidad administrativa resarcitoria y las } \\
\text { disciplinarias con algún riesgo de prescripción u otra situación que se } \\
\text { considere grave, para que actúe conforme a sus atribuciones. Esta } \\
\text { situación será mencionada en el informe de auditoría. }\end{array}$ \\
\hline 17 & Supervisor de auditoría & $\begin{array}{l}\text { Registra las observaciones con presunta responsabilidad } \\
\text { administrativa resarcitoria y las disciplinarias en el sistema } \\
\text { correspondiente. }\end{array}$ \\
\hline 18 & Supervisor de auditoría & $\begin{array}{l}\text { Recibe cédulas y en su caso efectúa correcciones, dejando evidencia } \\
\text { de la supervisión efectuada y entrega cédulas de observaciones al } \\
\text { Ente para sus comentarios. }\end{array}$ \\
\hline 19 & $\begin{array}{l}\text { Área Auditada de la Dependencia } \\
\text { o Entidad }\end{array}$ & $\begin{array}{l}\text { Recibe las cédulas de observaciones y presenta información y } \\
\text { aclaraciones. }\end{array}$ \\
\hline 20 & Supervisor de auditoría & $\begin{array}{l}\text { Analiza conjuntamente con el auditor las respuestas del Ente y } \\
\text { determina su razonabilidad, en su caso, instruye la aplicación de } \\
\text { procedimientos adicionales o solventa la observación, dejando } \\
\text { evidencia de tal hecho. }\end{array}$ \\
\hline
\end{tabular}


Tabla 6. Continuación...

\begin{tabular}{|c|c|c|}
\hline & Persona Responsable & Actividades \\
\hline & Inicio del procedimiento & \\
\hline 21 & Auditor & $\begin{array}{l}\text { Elabora informe de auditoría, considerando que la auditoría al } \\
\text { desempeño es propositiva y que su intención final es coadyuvar a que } \\
\text { se logren los objetivos del Ente, cuida la redacción, la claridad en la } \\
\text { problemática detectada y en la sugerencia, asimismo, incluye en los } \\
\text { informes temas de importancia y sin ambigüedad e integra expediente } \\
\text { de auditoría cruzado e indexado conforme a índice para la integración } \\
\text { de expedientes de auditoría y elabora cédula de supervisión de } \\
\text { auditoría. }\end{array}$ \\
\hline 22 & Supervisor de auditoría & $\begin{array}{l}\text { Verifica informe de auditoría, para ello: } \\
\text { - Revisa el cumplimiento del programa específico de auditoría y el } \\
\text { avance de la revisión en el cronograma de actividades. } \\
\text { - Determina el orden de presentación de los resultados por tipo de } \\
\text { observación, incluyendo todas las observaciones detectadas. } \\
\text { - Verifica que el informe cuente con las características de calidad, } \\
\text { oportunidad, integridad, competencia, relevancia, objetividad, } \\
\text { convicción, claridad y utilidad. Asimismo, verifica la congruencia de } \\
\text { las observaciones determinadas con las acciones de mejora a } \\
\text { implementar y recomendaciones. }\end{array}$ \\
\hline 23 & Titular del OIC & $\begin{array}{l}\text { Revisa y autoriza informe de auditoría y cédula de supervisión de } \\
\text { auditoría. }\end{array}$ \\
\hline 24 & Titular del OIC & $\begin{array}{l}\text { Se constituye en las oficinas del Ente, en la fecha programada para la } \\
\text { reunión formal y da a conocer el informe de auditoría a los Servidores } \\
\text { Públicos involucrados en el proceso sujeto a revisión, así como los } \\
\text { responsables de la implementación de las acciones de mejora que en } \\
\text { su caso se hayan detectado y se levanta acta de cierre de revisión } \\
\text { documental. }\end{array}$ \\
\hline
\end{tabular}


Tabla 6. Continuación...

\begin{tabular}{|c|l|l|}
\hline \multicolumn{1}{|c|}{ Persona Responsable } & \multicolumn{1}{|c|}{ Actividades } \\
\hline 25 & Inicio del procedimiento & $\begin{array}{l}\text { Elabora oficio de envío de informe de auditoría, y lo entrega al } \\
\text { Titular del Ente y demás autoridades correspondientes, obteniendo el } \\
\text { acuse respectivo. }\end{array}$ \\
\hline 26 & Supervisor de auditoría & $\begin{array}{l}\text { Registra resultados de la auditoría en el sistema correspondiente, } \\
\text { dando de alta todas las observaciones incluidas en el informe de } \\
\text { auditoría, así como los montos auditados. }\end{array}$ \\
\hline 27 & Titular del Área Auditada & $\begin{array}{l}\text { Recibe informe de auditoría y procede a la atención de las } \\
\text { observaciones. }\end{array}$ \\
\hline 29 & Supervisor de auditoría y Auditor & $\begin{array}{l}\text { Recibe oficio del Ente y soporte documental de la solventación de } \\
\text { observaciones, analiza y elabora cédula de seguimiento, ya sea para } \\
\text { solventación total o parcial y envía al Ente el oficio de resultados de } \\
\text { seguimiento e integra toda esta información al expediente de } \\
\text { auditoría. }\end{array}$ \\
\hline & Fin del procedimiento & $\begin{array}{l}\text { Archiva expediente de auditoría conforme a la normatividad } \\
\text { aplicable, previa instrucción de su superior inmediato. }\end{array}$ \\
\hline integrante del equipo de auditoría.
\end{tabular}

Fuente: Programa de Trabajo tomado del curso virtual “Seminario de Auditoría Gubernamental”, el cual fue diseñado por el Secretario de la Contraloría y Modernización Administrativa del Gobierno del Estado de Durango, procediendo a una modificación para adaptarlo a las necesidades de la presente investigación. 


\section{Referencias:}

\section{Referencias de libros:}

Arens, A; Randal E; Mark, B (2007). Auditoría un enfoque integral, México, Editorial Pearson, decimoprimera edición, p. 301

Arrollo, Francisco. Estudio sociológico jurídico sobre el delito de fraude. Editorial UNAM, 1era edición, México, 1962

Bernal, C. (2010). Metodología de la Investigación. Colombia, Ed. Pearson (p. 60, 113)

Blanco Luna, Yanel. Normas y Procedimientos de la auditoría Integral. Ecoe Ediciones, 1era edición, Colombia, 2004

De la Peña Gutiérrez, Alberto. Auditoría un enfoque práctico. Editorial Paraninfo, 1era edición, España, 2011.

Instituto Mexicano de Contadores Públicos. Auditoría a las Cuentas Públicas, Ed CeMPro, 1era edición, México, 2004

\section{Referencias base de datos:}

Funnell, W. \& Wade, M. (2012) Negotiating the credibility of performance auditing. Critical Perspectives on Accounting 23 (2012) 434- 450 (p. 434)

Hossain, S. (2010). From Project Audit to Performance Audit: Evolution of Performance Auditing. College of Business and Economics, the Australian National University, Canberra, ACT 0200 (p. 20)

Iatco, C., Ignat, G., Ungureanu, G. \& Athes, H. (2014). Aspects regarding the principles of performance audit. Lucrări Ştiinţifice - vol. 57 (2) 2014, seria Agronomie (p.253)

Jarquin, J, Molina, E. \& Roseth, B. (2018). Auditoría de desempeño para una mejor gestión pública en América Latina y el Caribe. Banco Interamericano de Desarrollo. Documento para discusión IDB-DP-587.
Leon, E., \& Lagunas, S. (2017). Auditoría Forense: Conceptualizaciones y adopción en América Latina. Revista Internacional, 3(3), 48-76. Recuperado de https://recai.uaemex.mx/article/view/9268/8377

McCrae, M. \& Vada, H. (1997. Performance audit scope and the independence of the Australian commonwealth auditor-general.

Financial Accountability\&Management,13(3), August 1997, 0267-4424 (p.203)

Raudla, R., Taro, K., Agu, Ch. \& Douglas, J. (2016). The Impact of Performance Audit on Public Sector Organizations: The Case of Estonia. Public Organiz Rev (2016) 16:217-233 (p. 219). DOI 10.1007/s11115015-0308-0

Silveira, R. (2019). A new classification for the results of the performance audit follow up. Rio de Janeiro 53(1):23-44 Jan. - Feb. 2019 (p.23)

Voiculescu, S. \& Tătaru, V. (2019). The performance audit. the experience of other supreme audit institutions. University, The Faculty of Economic Studies, Bucuresti, str. Govora, nr. 2, bl. 74, sc. 4, et 1, ap 50. (p. 1521)

\section{Referencias páginas web:}

Curso virtual "Seminario de Auditoría Gubernamental", diseñado por el Secretario de la Contraloría y Modernización Administrativa del Gobierno del Estado de Durango

Portal de internet de la Organización Internacional de las Entidades Fiscalizadoras Superiores (INTOSAI). Principios Fundamentales de la Auditoría de Desempeño. Recuperado el 19 de junio de 2019 de:

http://www.cytg2.nl.gob.mx/SNF/documentos/Modulo_ V/Modulo\%20V/ISSAI_300_Aud_Desempeno.pdf

Portal de internet de la Universidad de Sonora: www.bidi.uson.mx 


\section{Referencias legales:}

Portal de internet Congreso del Estado de Sonora.

Constitución Política del Estado de Sonora. Recuperado el 19 de junio de 2019 de:

http://www.congresoson.gob.mx:81/Content/Doc_leyes/ doc_446.pdf

Ley Orgánica del Poder ejecutivo del Estado de Sonora. Recuperado el 19 de junio de 2019 de:

Ley de Fiscalización Superior del Estado de Sonora. Recuperado el 19 de junio de 2019 de:

Ley de Gobierno y Administración Municipal. Recuperado el 19 de junio de 2019 de:

http://www.congresoson.gob.mx/transparencia/leyes

Portal de internet Cámara de Diputados del H. Congreso de la Unión

Constitución Política de los Estados Unidos Mexicanos. Recuperado el 19 de junio de 2019 de: http://www.diputados.gob.mx/LeyesBiblio/htm/1.htm

Ley General de Contabilidad Gubernamental. Recuperado el 19 de junio de 2019 de: http://www.diputados.gob.mx/LeyesBiblio/pdf/LGCG_ 300118.pdf

Ley General del Sistema Nacional Anticorrupción (2019). Recuperado el 13 de junio de 2019 de: http://www.diputados.gob.mx/LeyesBiblio/pdf/LGSN A.pdf

Portal de internet Supremo Tribunal de Justicia de Sonora.

Ley del Sistema Estatal Anticorrupción (2017). Recuperado el 13 de junio de 2019 de: http://www.stjsonora.gob.mx/reformas/Reformas11051 7-2.PDF

Portal de internet Secretaria de Hacienda y Crédito Público.
Acuerdo por el que se establecen las disposiciones generales del Sistema de Evaluación del Desempeño (2019). Recuperado el 13 de junio de 2019 de:

https://www.gob.mx/cms/uploads/attachment/file/15442 7/acuerdo_sed.pdf

Portal de Internet del Consejo Nacional de Armonización Contable (CONAC). Recuperado el 13 de junio de 2019 de: https://www.conac.gob.mx/

Portal de internet Auditoría Superior de la Federación. Recuperado el 13 de junio de 2019 de: https://www.asf.gob.mx/Default/Index

Portal de internet Secretaría de la Función Pública. Recuperado el 13 de junio de 2019 de:

http://www.funcionpublica.gob.mx/index.php/unidadesadministrativas/unidad-de-auditoriagubernamental/guias-de-auditoria.html

https://www.asf.gob.mx/Section/53_Tipos_de_auditorias _desarrolladas

Portal de internet Secretaría de la Contraloría General. Sistema de Control Interno. Comités de Control y Desempeño Institucional COCODI (2019). Recuperado el 13 de junio de 2019 de:

http://contraloria.sonora.gob.mx/gubernamental/sistemade-control-interno-institucional.html

Portal de internet Instituto Superior de Auditoría y Fiscalización. Programa Anual de Auditorías 201820189. Recuperado el 13 de junio de 2019 de:

https://intranet.isaf.gob.mx/Publico/Documento/3144

Portal de Internet ayuntamiento de Hermosillo http://www.hermosillo.gob.mx/portaltransparencia/marc o_legal.aspx

http://www.hermosillo.gob.mx/entidades/?id=10 